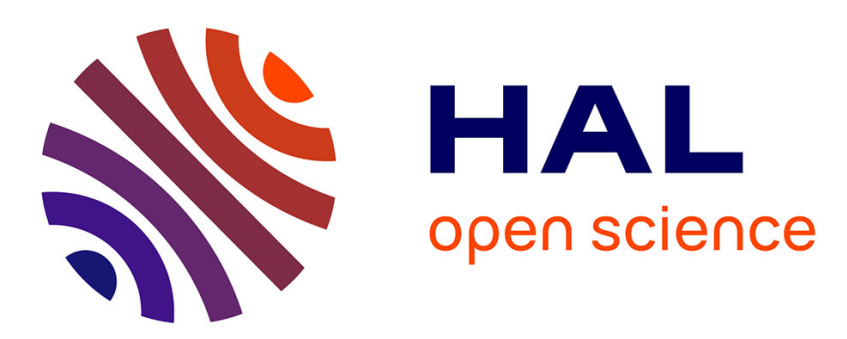

\title{
A MENAGERIE OF INTERFACE STRUCTURES IN COPOLYMER SYSTEMS
}

\author{
E. Thomas, J. Reffner, J. Bellare
}

\section{To cite this version:}

E. Thomas, J. Reffner, J. Bellare. A MENAGERIE OF INTERFACE STRUCTURES IN COPOLYMER SYSTEMS. Journal de Physique Colloques, 1990, 51 (C7), pp.C7-363-C7-374. 10.1051/jphyscol:1990736 . jpa-00231135

\section{HAL Id: jpa-00231135 https://hal.science/jpa-00231135}

Submitted on 1 Jan 1990

HAL is a multi-disciplinary open access archive for the deposit and dissemination of scientific research documents, whether they are published or not. The documents may come from teaching and research institutions in France or abroad, or from public or private research centers.
L'archive ouverte pluridisciplinaire HAL, est destinée au dépôt et à la diffusion de documents scientifiques de niveau recherche, publiés ou non, émanant des établissements d'enseignement et de recherche français ou étrangers, des laboratoires publics ou privés. 


\title{
A MENAGERIE OF INTERFACE STRUCTURES IN COPOLYMER SYSTEMS
}

\author{
E.L. THOMAS, J.R. REFFNER* and J. BELLARE** \\ Department of Materials Science and Engineering, Massachusetts \\ Institute of Technology, Cambridge MA 02139, U.S.A. \\ * Department of Polymer Science and Engineering. University of \\ Massachusetts, Amherst MA 01003, U.S.A. \\ ** Department of Chemical Engineering, Indian Institute of Technology, \\ Bombay 400 076. India
}

\begin{abstract}
Résumé_Les macromolecules de copolymere dibloc peuvent etre considérées comme amphophiles. De telles molecules s'auto-assemblent instantanement sous forme d'un grand nombre de structures qui presentent des surfaces de courbure moyenne a peu pres constante separant les composants du materiau. Les transitions ordre-desordre dans les materiaux non traités resultent en des structures de micro-domaines ordonnés formes de spheres $\mathrm{BCC}$, de cylindres groupés hexagonalement et de lamelles empilées. De plus la structure double diamant bicontinu ordonne (OBDD) se trouve aussi apparaitre sous forme de phase cubique entre les morphologies cylindriques et lamellaires. La structure de l'interface de l'OBDD ressemble a une famille de surfaces de courbure moyenne constante récemment découvertes qui incluent la surface minimale D de Schwarz. Une autre structure d'interface qui approxime la Premiere Surface de Scherk est une rotation de 90 degrés de la frontiere entre les grains de lamelles. Le melange d'un copolymere dibloc avec un homopolymere fournit un systeme binaire aux usages variés qui presente une concentration micellaire critique, des micelles spheriques et cylindriques, des vesicles lamellaires ainsi que des transitions ordoneés dependant de la composition. La pénétration du homopolymere dans un coté de la region proche de l'interface joue un role dans les variations observées de la courbure de l'interface dans les melanges aussi bien que dans le copolymere dibloc pur. Des geometries confinantes telles que les surfaces externes, les films fins et les micro-gouttelettes peuvent influencer les structures de l'interface. Il a eté montré que des structures chaotiques discontinues ainsi que paralleles a la surface telles que des coques spheriques concentriques apparaissent dans les micro-gouttelettes d'un copolymere dibloc qui forment des microdomaines lamellaires dans le materiau.
\end{abstract}

Abstract-Diblock copolymer macromolecules may be viewed as giant amphiphiles. Such molecules readily self-assemble into a wide variety of structures which exhibit intermaterial dividing surfaces of approximately constant mean curvature. Order-disorder transitions in bulk materials result in ordered microdomain structures of BCC spheres, hexagonally packed cylinders and stacked lamella. An additional structure, the ordered bicontinuous doublediamond (OBDD) is also shown to occur as a cubic phase between the cylindrical and lamellar morphologies. The interface structure of the OBDD resembles a recently discovered family of constant mean curvature surfaces which include the Schwarz D minimal surface. Another interface structure which approximates Scherk's First Surface is a 90 twist boundary between grains of lamella. Blending of diblock copolymer with homopolymer provides a versatile binary system which displays a critical micelle concentration, spherical and cylindrical micelles, lamellar vesicles as well as ordering transitions depending on composition. The penetration of homopolymer into one side of the near-interface region accounts for the observed variations in interface curvature in blends from that of the pure diblock. Confining geometries, such as external surfaces, thin films and microdroplets can influence interface structures. Chaotic bicontinuous as well as parallel surface structures such as concentric spherical shells are shown to occur in microdroplets of a diblock copolymer which forms lamellar microdomains in the bulk.

\footnotetext{
${ }^{1}$ Department of Polymer Science and Engineering, University of Massachusetts, Amherst, Massachusetts 01002 .
}

2Department of Chemical Engineering, Indian Institute of Technology, Bombay, India 400076 


\section{INTRODUCTION}

A goal in polymer science is to understand how macromolecules self assemble. The macromolecules of interest encompass a very wide variety of molecular microstructures and types of intra- and inter- molecular interactions. In this paper we report work on the types of geometrical arrangements formed via self assembly in simple noncrystalline systems comprised of either a diblock copolymer or a blend of diblock copolymer with homopolymer.

The copolymer is made of two blocks, as shown below:

$$
\text { AAAA...AA - BBBB...BB or simply } A_{n} B_{m}
$$

where typically in our studies $A$ is polystyrene and $B$ is polyisoprene or polybutadiene. Values of the block degree of polymerization $(n, m)$ are on the order of $10^{2}-10^{4}$. Because the two segments normally have an unfavorable energy of interaction, they mutually aggregate into A-rich and B-rich regions such that $\mathrm{A}-\mathrm{B}$ contacts are minimized (see figure 1). The key feature of a covalent link between the two types of segments forces the scale of aggregation to be on the radius of gyration of a block (typically $100 \AA$ ), moreover at equilibrium, the aggregates form periodic structures with the covalent link junction between the $A$ and $B$ segments confined to the interface.

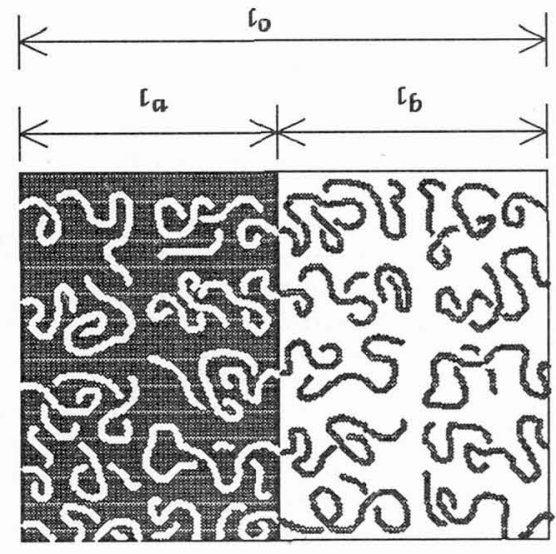

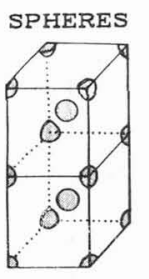

0. $21 \%$

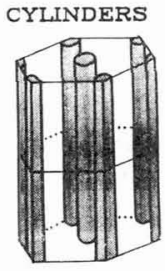

$21-28 \%$

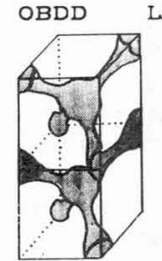

$28-33 \%$

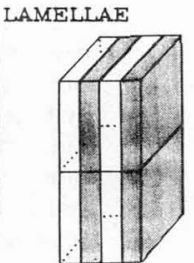

$33-50 \%$

B

A

Fig. 1 a) Schematic of polymer chains in microphase separated state.

b) Microdomain morphology as a function of volume fraction minority phase. Composition windows refer to the polyisoprene (minority) polystyrene system. 
Anionic synthesis techniques can readily produce linear, branched, star and multiblock chain architectures with narrow distributions of composition, chain length and topology [1]. The prime variables are the asymmetry of the chain (ratio of number of A segments to B segments), the total length of the chain and the topology (e.g. linear, branched, or star). At high temperatures, entropy favors a mixed state whereas at lower temperatures, an ordering transition occurs due to self-aggregation. For example at $n / m=1$ (for chains comprised of monomers of approximately equal volume), a lamellar structure forms, (i.e. a series of flat, parallel, alternating $A$ and $B$ domains as illustrated in figure 1). There are two general theoretical approaches for the ordering transition, the so called weak segregation theory of Leibler [2] which is valid near the transition and the strong segregation theory first given by Helfand [3] which predicts the type of ordered domains formed far from the ordering transition. The key variables are the total degree of polymerization, $N(N=$ $\mathrm{n}+\mathrm{m}), \mathrm{X}$, the Flory parameter which characterizes the A - B segment-segment interaction $(X \sim 1 / T)$ and the composition, $f=m / n+m$. Figure 2 shows the dependence of $\mathrm{XN}$ vs. $\mathrm{f}$ for both the weak segregation region ( $\mathrm{XN}$ on the order of 10 ) and the strong segregation region ( $\mathrm{XN}>100$ ). For weakly segregated systems near the orderdisorder transition, the intermaterial boundary is diffuse, with a breadth on the same size scale as the domains: The morphology diagram is strongly dependent on $\mathrm{XN}$ with the stability of the homogeneously mixed (disordered) phase increasing offsymmetry. One sees that for strong segregation, the ordered morphology depends only on composition with BCC packed spherical micelles succeeded by hexagonally packed cylinders and finally alternating lamellar domains with increasing volume fraction of the minority block. In the strong segregation regime, the interfacial region is narrow compared to the scale of the domain size, thus treating the PS/PI interface as a thin intermaterial surface is quite reasonable. An additional strong segregation limit domain morphology, the ordered bicontinuous double diamond

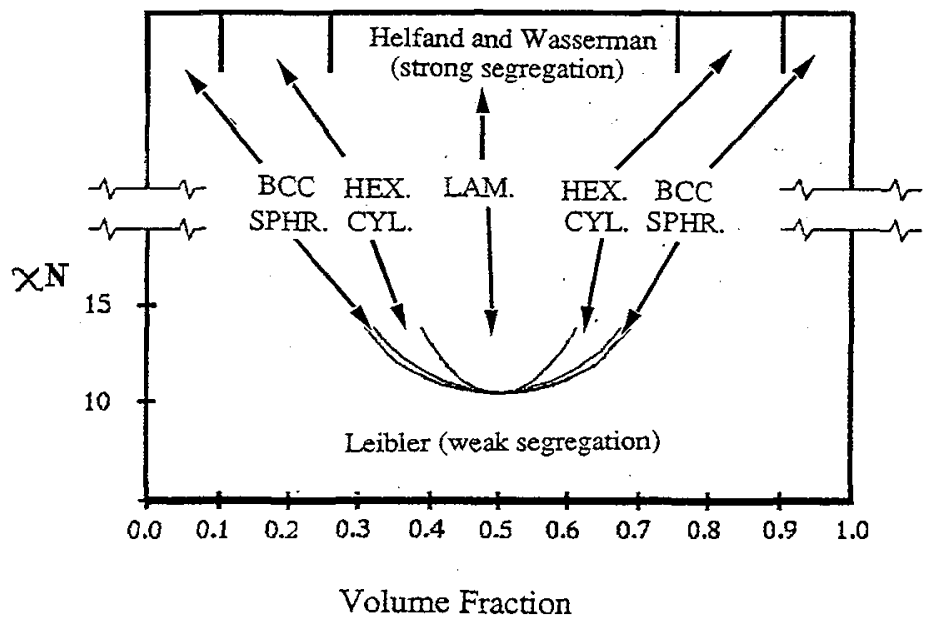

Fig. 2 Diblock copolymer morphology diagram showing weak segregation regime (bottom) and strong segregation regime (top). 
(OBDD) was first discovered in star diblock materials [4]. It was subsequently also found in linear diblocks [5], in binary blends of diblock copolymer and homopolymer [6] and in diblock-diblock blends [7]. The four basic types of domain structures are shown schematically in figure $1 \mathrm{~b}$.

The OBDD domain morphology occurs in a composition range of 28-33\% and 62-66 volume \% polystyrene in the polystyrene-co-polydiene system. In the OBDD structure, one phase (e.g. polyisoprene) resides in two intertwined but distinct labyrinthine networks, each exhibiting diamond cubic symmetry and the other phase (e.g. polystyrene) resides in the continuous matrix between the two diamond channels. The space group is $\mathrm{Pn} 3 \mathrm{~m}$ and the matrix is bisected by a connected triply periodic minimal surface (Schwarz's D surface) [8]. Figure 3a shows a computer graphics representation of the structure. The OBDD morphology appears as a cubic phase intermediate in composition between the lamellar and cylindrical phases in direct analogy to the sequential structures observed in small molecule amphiphilic systems.

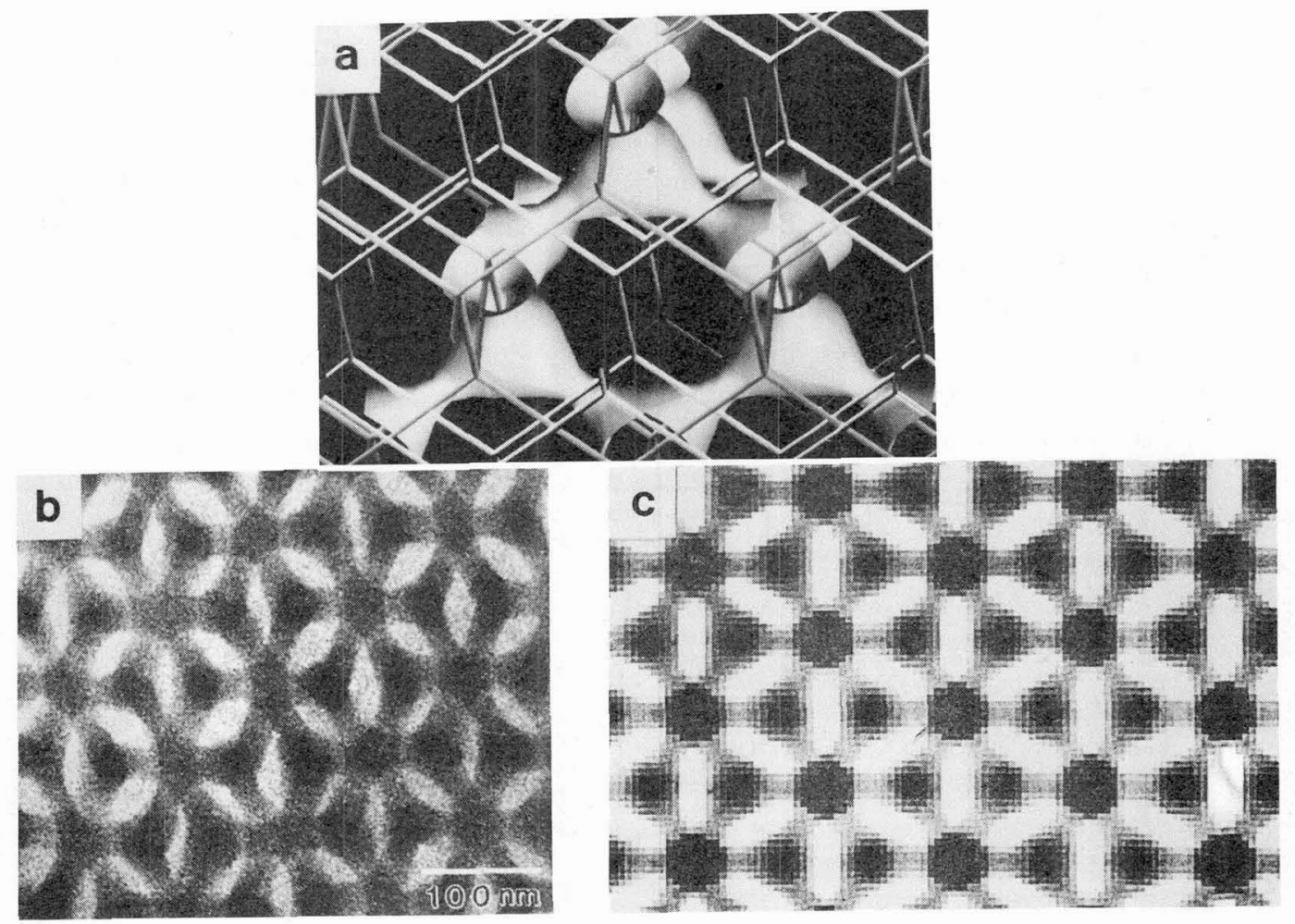

Fig. 3 a). Computer-generated image of a triply periodic surface of constant mean curvature (D. M. Anderson).

b). Bright field TEM image of the microdomain morphology of a PS/PI block copolymer.

c). Computer-simulated [111] projection of the model structure whose PS/PI interface is figure $3 \mathbf{a}$. 
The intermaterial A-B interface in all four types of domain structure exhibits approximately constant mean curvature. Surfaces of constant mean curvature arise naturally in mathematics as the consequence of the minimization of interfacial area at fixed volume fraction (composition). An important additional feature entering into the determination of the precise domain interface structure is the need to accomodate the chains within the regions delineated by the intermaterial surfaces. Since polymer chains of high degree of polymerization approximate three dimensional random walks, the characteristic lengths of the respective domains $\mathbb{L}_{\mathrm{A}}$ and $\mathrm{L}_{\mathrm{B}}$, should scale with $\mathrm{N}^{1 / 2}$. Thermodynamic equilibrium structures in block copolymer systems must thus minimize both the interfacial area and chain deformation under the constraints of uniform segmental density and fixed volume fraction.

Experimentally, the domain size scales as $\mathrm{N}^{2 / 3}$ so that the chains are actually stretched in a direction normal to the intermaterial dividing surface. This additional feature of chain deformation may therefore alter the precise interfacial curvature and result in surfaces of other than constant mean curvature. Transmission electron microscopy (TEM) data suggest that constant mean curvature surfaces are quite good approximations to the actual intermaterial dividing surfaces.

The sequence of domain geometries: spheres, cylinders, double diamond and lamellae show a steadily decreasing interfacial mean curvature with decreasing compositional asymmetry. In simple amphiphilic systems, interface curvature can be anticipated by the ratio of the surfactant volume $\mathbf{v}$ to the product of the head group area $\mathbf{a}$ and the chain length $\mathbf{l}$ (i.e. v/al). In pure block copolymer systems it is the ratio of the volume of the $A$ segment to that of the $B$ segment $V_{A} / V_{B}$ which influences interface curvature. Additionally, the penetration of low molecular weight homopolymer into the near-interface region allows further control of the interface curvature in diblock copolymer/homopolymer blends.

\section{EXPERIMENTAL METHODS}

Structural characterization of polymeric amphiphilic systems is rather straightforward owing to the large length scale (determined by the synthesis of a particular molecular weight material) and long relaxation times of polymexic liquids. TEM and small angle x-ray scattering (SAXS) are useful for determination of the domain geometry by direct inspection and precise statistically reliable determination of domain size and spacing respectively. In order to visualize the interfaces, a selective heavy atom stain $\left(\mathrm{OsO}_{4}\right)$ is used to preferentially oxidize the double bonds in the polydiene component and thus provide mass thickness contrast for bright field electron micrographs. The inherent electron density difference between the two unstained components is sufficient for a strong SAXS signal. 
Bulk samples are prepared by slow solvent casting from toluene (a nonpreferrential solvent) followed by a one week anneal above the glass transition temperature of the polystyrene but below the order/disorder transition and low enough to prevent chemical degradation. After annealing, samples are quenched to room temperature. Thus, while the samples are examined as solids, the microdomain structures are characteristic of the liquid/liquid phase separated state at the annealing temperature.

Polymer particles, in the size range of $100-5,000 \AA$ in diameter, referred to as 'microdroplets', are produced by an aerosol technique [9]. A dilute polymer solution $(0.1 \%)$ was nebulized to form small droplets that are heated to remove solvent and to anneal, stained to provide contrast and deposited onto TEM grids for observation.

Three dimensional model interface structures are examined by rendering in perspective. Detailed comparison of candidate models to experimental data requires the production of two dimensional simulated images based on projections through finite sections of the three dimensional structures. It is useful to catalog characteristic "signature" images of the complex candidate structures to aid in pattern recognition and quantitative matches. We are thus interested in computing two dimensional projections from various candidate three dimensional intermaterial dividing surfaces. Three approaches have been used to represent the dividing surfaces: traditional coordinate systems, finite element representations and tesselations adapted to complex line integration schemes [10]. Input data is the surface of constant mean curvature, its orientation and the location of parallel upper and lower truncating planes representing the specimen thickness determined by microtomy. Experimental micrographs can be simulated by sending rays through the three dimensional theoretical structure and calculating the resulting two dimensional projection at each pixel. The projected pixel intensity is determined by testing a large number of evenly spaced points along each ray, finding which material they lie in and taking the projected intensity at a given pixel to be a linear fraction of points lying within a particular phase (intensity of zero and one for unstained PS and stained polydiene phases respectively). An M $\mathrm{M}$ array of theoretical intensities based on a structural model determined by some intermaterial dividing surface is compared to a corresponding array of experimental pixel intensities obtained by microdensitometry of an electron micrograph. The quality of fit between the experimental array and the theoretical array can be quantified by a root mean square norm over all pixels in a given region of interest (e.g. a unit cell of the projected structure). 


\section{RESULTS AND DISCUSSION}

\section{GRAIN BOUNDARY STRUCTURE}

In addition to the microdomain structure itself, it is of interest to understand the grain superstructure of the microdomains. The specific structure of the boundary region between grains is of importance for physical properties such as modulus and permeability that depend on the continuity of the phases across such boundaries. The simplest case is that of the lamellar microdomain structure. A situation that is analogous to a $90^{\circ}$ twist boundary in crystals occurs in polymeric systems when the normals of two adjacent sets of lamellae are orthogonal. In essence, the problem is to smoothly join the two sets of evenly spaced planes that meet orthogonally. The solution to this problem is a minimal surface, called Scherk's First Surface [11], shown in perspective in figure 4a. The interface where the two sets of lamellae come together consists of a doubly periodic array of saddle-surface regions [12]. The characteristic saddle shape is best seen in projection when viewed along one of the normals to the lamellae (see projection simulation in figure $4 \mathrm{~b}$ ). Figure $4 \mathrm{c}$ shows an electron micrograph of a region of a $\mathrm{PS} / \mathrm{PB}$ film in which the lamellae undergo a $90^{\circ}$ twist reorientation. The image corresponds well with the simulation for this and other projected views, strongly suggesting that the actual boundary interface between the two grains approximates Scherk's minimal surface, which affords both optimal phase continuity and minimizes unfavorable $\mathrm{A} / \mathrm{B}$ segment-segment contacts between phases.

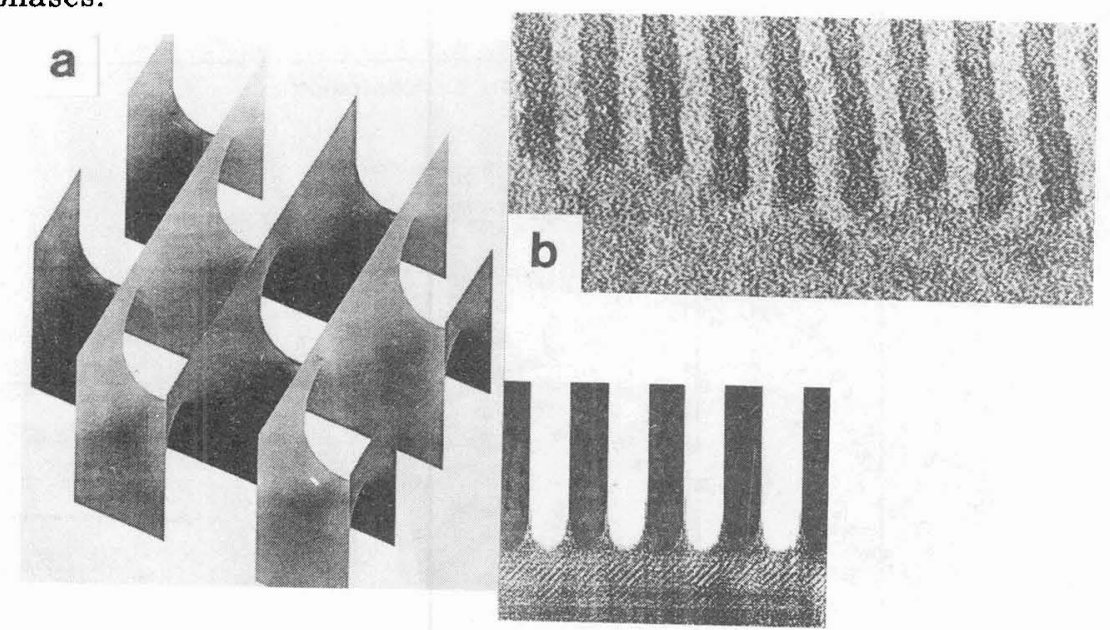

Fig. 4 a). Computer-generated image of Scherk's first minimal surface. (J.T. Hoffman).

b). Bright field TEM image of the lamellar microdomain morphology of a PS/PB block copolymer.

c). Computer-simulated projection of a structure whose PS/PB interface is figure $4 a$. 


\section{BINARY BLENDS OF DIBLOCKS AND HOMOPOLYMERS}

Figure 5a depicts the sequence of morphologies encountered with increasing diblock content in a binary blend of diblock copolymer and polystyrene homopolymer. At very low diblock content, the system is a homogeneous solution. Above a certain concentration (the critical micelle concentration), it becomes favorable for the polydiene segments of the diblock chains to aggregate, here shown forming spherical micelles in the PS homopolymer matrix (see figure $5 \mathrm{~b}$ ). Such micelles consist of a core region of essentially pure polydiene material surrounded by a corona of the attached PS chains swollen with the PS homopolymer. Depending on molecular parameters such as composition and molecular weight, as well as temperature, worm-like cylindrical micelles (figure 5c) or vesicular-like lamellar bilayers (figure 5d) may form instead of spherical micelles [13].

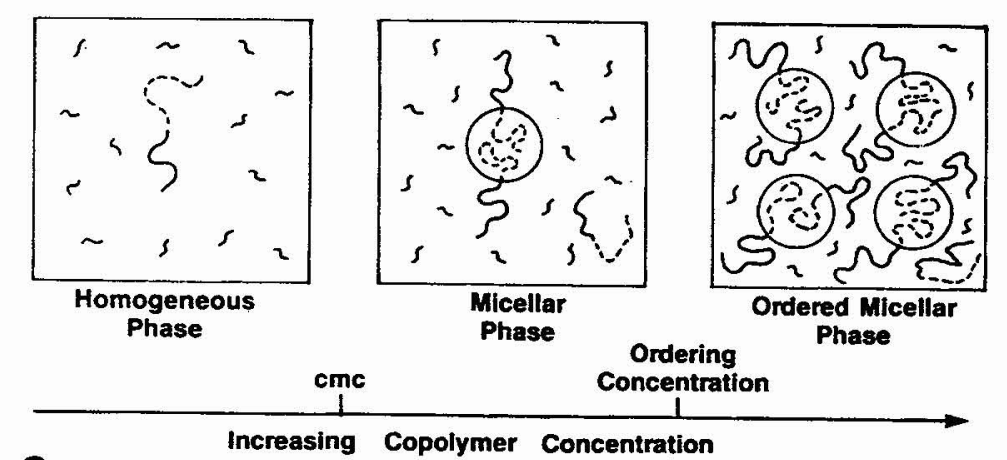

a

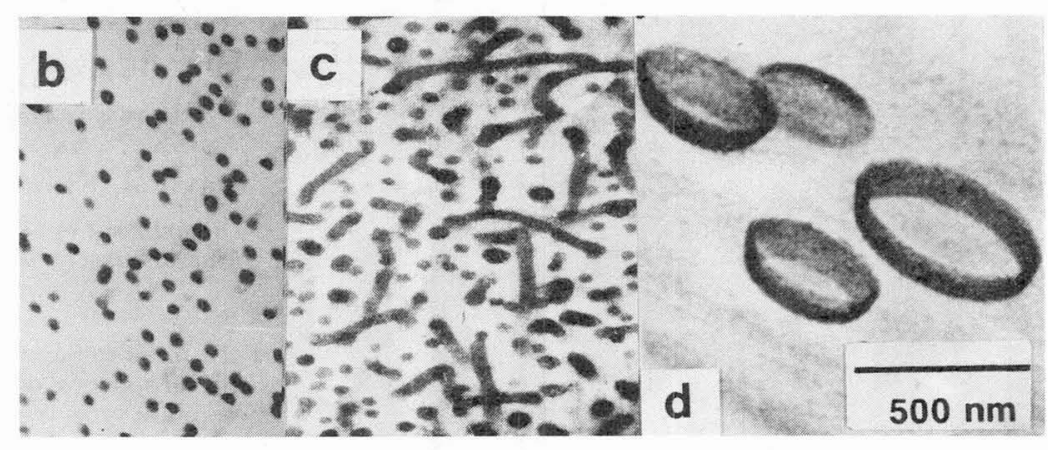

Fig. 5 a). Schematic of various morphologies of a dilute diblock/homopolymer blend.

Bright field TEM images of spherical micelles (b), cylindrical micelles (c) and lamellar vesicles (d). 
Studies involving TEM and SAXS using the Percus-Yevick hard sphere fluid model to obtain the interparticle interference contribution to the scattered intensity have allowed thorough characterization of the structure of spherical micelles as a function of molecular parameters [14]. Recently we have extended our blends investigations into the more concentrated diblock regime and encountered a series of ordered microdomain structures entirely analogous to those occurring in water/surfactant systems [15]. In particular, we find ordered spherical micelles on a primitive cubic lattice, cylindrical micelles on a hexagonal lattice, the cubic double diamond phase, and a swollen lamellar phase (see figures 6a-d). The ordering of spherical micelles is interesting since micelles of pure diblock copolymers are arranged on a BCC lattice [16, 17], whereas for binary blends, the packing is primitive cubic [14]. This is thought to be due to the differing requirements of the outer block chains which must stretch to uniformly fill the space between the domains. The BCC arrangement minimizes this stretching, whereas in the blend the mobile homopolymer chains afford an additional degree of freedom in filling space [17]. For low molecular weight homopolymers such as we have studied, Leibler and Pincus predict a strictly repulsive intermicelle potential [1.8]. However, when the molecular weight of the homopolymer is sufficiently high, it has been observed that the interaction of micelles with other micelles, interfaces and external surfaces is attractive [19].

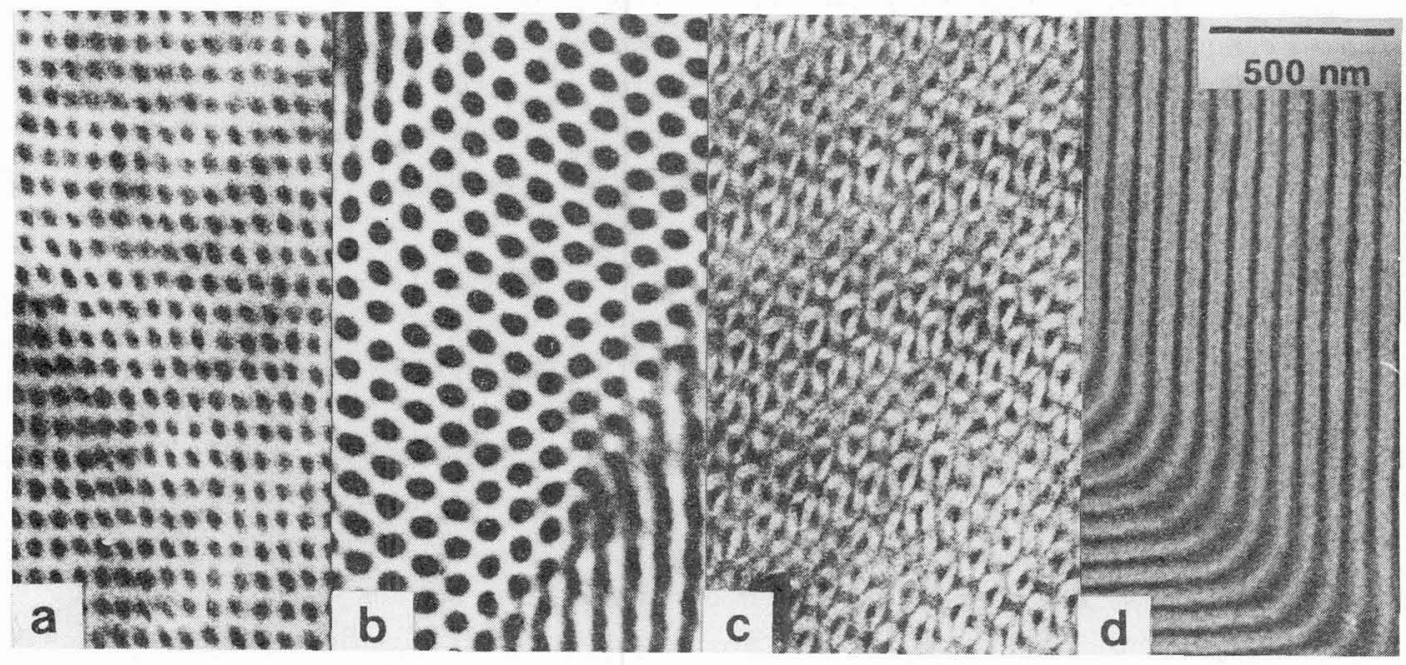

Fig. 6 TEM micrographs of a series of blends of PS/PI diblock with PS homopolymer.

a). Spherical micelles on a primitive cubic lattice at $70 \%$ homopolymer content.

b). Cylindrical micelles on a hexagonal lattice at 50\% homopolymer content.

c). Double diamond structure at $30 \%$ homopolymer content.

d). Swollen lamellar structure at $10 \%$ homopolymer content. 
The composition ranges over which the various ordered microdomain structures occur is nearly the same for both the diblock materials and the diblock/homopolymer blends [13]. The mean curvature of the intermaterial dividing surfaces decreases systematically with change in the morphology from spheres to cylinders to double diamond to lamella. An interesting aspect of polymeric systems is the ability to manipulate structure at constant composition by simple variation of solvent (or equivalently the diblock) molecular weight. This degree of freedom is not, of course, available with traditional amphiphilic systems. The penetration of the homopolymer solvent into the corona region serves to alter the preferred interface curvature. The degree and extent of penetration depends inversely on the molecular weight of the homopolymer. As the homopolymer penetrates into the near-interfacial region, the interface area per chain increases which causes the $B$ domain to decrease its thickness in order to maintain uniform density. Thus preferential swelling on one side of the interface causes contraction on the other.

\section{MICRODOMAIN GEOMETRY IN MICRODROPLETS}

Lamellar diblock copolymers are similar to smectic liquid crystals and thus prefer textures that provide parallel surface structures. Sample compositions which exhibit ordered lamellae (equidistant parallel planes) in the bulk demonstrate two types of morphology when confined to microdroplets. Low molecular weight symmetric diblocks form concentric spherical shells (see figure 7a) of alternating components with the outer shell always composed of the lower surface tension component (polydiene block). This structure provides the lowest energy for lamellae since only a point singularity at the sphere center occurs.

For higher molecular weight diblocks, the bending energy which scales with $\mathrm{N}^{5 / 3}$ becomes excessive and a chaotic bicontinuous structure forms instead of parallel surfaces (see figure $7 \mathrm{~b}$ ). The intermaterial dividing surface now appears as a high genus labyrinthine structure. We speculate that this may be a block copolymer version of the "plumbers nightmare" structure (so called sponge phase). The intermaterial surface structures in the smallest droplets appear to be surface patches resembling the Schwarz D minimal surface. Indeed, it is not unreasonable to expect that portions of periodic minimal surface structures which subdivide the droplet into 50/50 volume fraction are candidates for the chaotic microdroplet morphologies of symmetric diblock composition.

Other bulk diblock copolymer microdomain morphologies are also influenced by the confining geometry of a microdroplet. For example, the ordered bicontinuous double diamond structure which is triply periodic is frustrated by the confining surface, and forms a polydiene outer shell with a concentric, continuous lacy network of polydiene in the droplet interior. The network appears to be made up of short cylindrical-like sections, not unlike a distorted version of the gyroid type networks proposed for oil/surfactant systems by Luzatti [20]. 

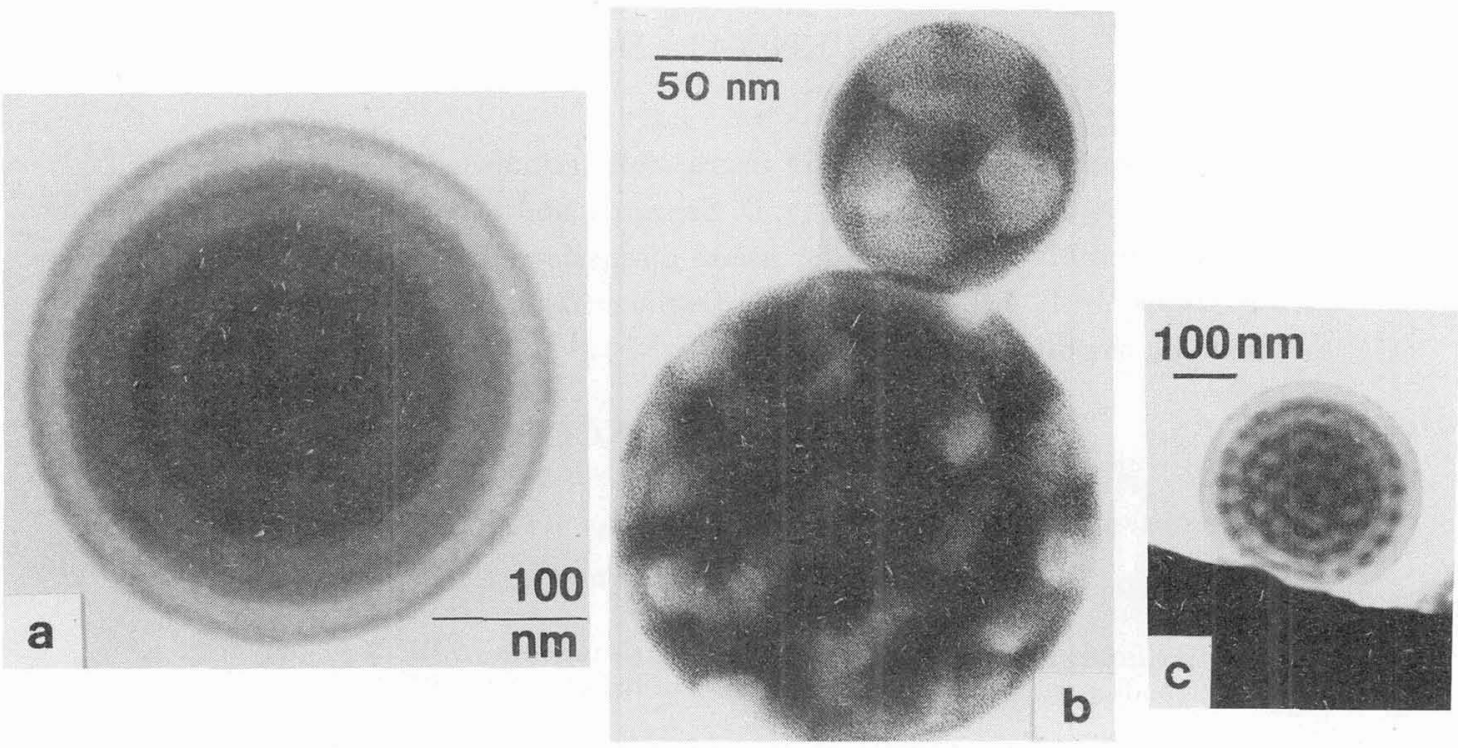

Fig. 7. TEM micrographs of diblock copolymer microdroplets.

a). A 20,000/20,000 PS/PB diblock copolymer forms concentric spherical shells.

b). An $80,000 / 80,000 \mathrm{PS} / \mathrm{PB}$ diblock copolymer exhibits a chaotic bicontinuous structure.

c). A $20,000 / 20,000 \mathrm{PS} / \mathrm{PB}$ diblock copolymer blended with $30 \% 17,000$ PS homopolymer which normally forms the double diamond structure in bulk exhibits a distorted PB network.

\section{SUMMARY}

Block copolymers are like giant amphiphiles and their self-assembly results in a wide variety of microdomain structures. Block copolymer/homopolymer blends exhibit essentially all the usual amphiphilic/solvent behavior: a critical micelle concentration, spherical and cylindrical micelles, lamellar vesicles, an ordered cubic lattice of spherical micelles, ordered cylinders on a hexagonal lattice, an ordered cubic phase (double diamond) and stacked lamellae. Frustration induced by highly curved confining surfaces induces chaotic bicontinuous structures of high genus in microdroplets. Because interfacial tension tends to minimize the area of the intermaterial dividing surfaces, we place emphasis on model candidate surfaces of constant mean curvature and minimal surfaces, which minimize area under volume fraction constraints and fixed boundary conditions respectively. We employ scattering and microscopy to elucidate structure. X-ray diffraction determines the space group and transmission electron microscopy allows direct visualization of the surface geometry. These techniques are combined with two dimensional computer simulation of images based on three dimensional theoretical surface structures. 


\section{ACKNOWLEDGEMENTS}

Financial support from and NSF is gratefully acknowledged. We thank Drs. D. Anderson, D. Gobran, D. Kinning, D. Schwark, and K. Winey; and Professor D. Hoffman and Mr. J. Hoffman for useful discussions and computer graphics. We also thank Dr. L. J. Fetters of Exxon Engineering and Research Company for providing the diblock copolymer samples.

\section{REFERENCES}

1. Fetters, L. J., J. Polym Sci C26, (1969), 1.

2. Leibler, L., Macromolecules $\underline{13}$, (1980), 1602.

3. Helfand, E., Wassermann, Z. R., Developments in Block Copolymers-I, Goodman, I. ed. Applied Science Publishers, NY, (1982).

4. Thomas, E. L., Alward, D. B., Kinning, D. J., Martin, D. C., Handlin, D. L. Fetters, L. J., Macromolecules 19, (1986), 2197.

5. Hasegawa, H., Tanaka, K. Yamasaki,K., Hashimoto, T., Macromolecules 20, (1987), 1651

6. Winey, K. I., Ph.D. Thesis, University of Massachusetts, 1991.

7. Schwark, D., MIT, private communication.

8. Schwarz, H. A., Gesamm. Math. Abh., Springer, Berlin, (1890).

9. Bellare, J. R., EMSA Proceedings, 47, (1989), 354.

10. Anderson, D. M., Bellare, J. R., Hoffman, J. T., Hoffman, D., Gunther, J., and Thomas, E. L., J. Coll. Interface Sci, to be published.

11. Scherk, H. F., J. R. Angew. Math 13, (1835), 185.

12. Thomas, E. L., Anderson, D. M., Henkee, C. S., and D. Hoffman, Nature $\underline{334}$, (1988), 598.

13. Kinning, D. J., Winey, K. I., and Thomas, E. L., Macromolecules 21, (1988), 3502.

14. Kinning, D. J., Fetters, L. J. and Thomas, E. L., J. Chem. Phys. 90, (1989), 5806.

15. Winey, K. I., and Thomas, E. L., MRS Proceedings 171, (1990), 225.

16. Bates, F. S., Cohen, R. E., and Berney, C. V., Macromolecules 15, (1982), 589.

17. Thomas, E. L., Kinning, D. J., Alward, D. B. and Henkee, C. S., Macromolecules 20, (1987), 2934.

18. Leibler, L., and Pincus, P. A., Macromolecules 17, (1984), 2922.

19. Schull, K., Winey, K. I., Kramer, E. J., and Thomas, E. L., Macromolecules, to be published.

20. Luzatti, V., Spegt, P. A., Nature 215, (1968), 701. 